\title{
Evaluation of cholinergic markers in Alzheimer's disease and in a model of cholinergic deficit
}

\author{
Francisco Javier Gil-Bea, Mónica García-Alloza, Jon Domínguez, \\ Beatriz Marcos, María Javier Ramírez* \\ Laboratory of Neuropharmacology, Department Pharmacology, School of Medicine, Center for Applied Medical Research (CIMA), \\ University of Navarra, Irunlarrea 1, 31008 Pamplona, Spain
}

Received 30 July 2004; received in revised form 21 October 2004; accepted 22 October 2004

\begin{abstract}
Cognitive deficits in neuropsychiatric disorders, such as Alzheimer's disease (AD), have been closely related to cholinergic deficits. We have compared different markers of cholinergic function to assess the best biomarker of cognitive deficits associated to cholinergic hypoactivity. In post-mortem frontal cortex from AD patients, acetylcholine (ACh) levels, cholinacetyltransferase (ChAT) and acetylcholinesterase (AChE) activity were all reduced compared to controls. Both ChAT and AChE activity showed a significant correlation with cognitive deficits. In the frontal cortex of rats with a selective cholinergic lesion, all cholinergic parameters measured (ACh levels, ChAT and AChE activities, "in vitro" and "in vivo" basal ACh release) were significantly reduced. AChE activity was associated to ChAT activity, and even more, to "in vivo" and "in vitro" basal ACh release. Quantification of AChE activity is performed by an easy and cheap method and therefore, these results suggest that determination of AChE activity may be used as an effective first step method to evaluate cholinergic deficits.
\end{abstract}

(C) 2004 Elsevier Ireland Ltd. All rights reserved.

Keywords: Acetylcholine (ACh); Acetylcholinesterase (AChE); Cholinacetyltransferase (ChAT); Human; Cognitive deficit; Rat

Cognitive deficits in Alzheimer's disease (AD) have been widely associated with dysfunction of the cholinergic system [10] consequent upon degeneration of cholinergic cell bodies in the basal forebrain [12]. Reflecting the loss of cholinergic innervation, reductions in acetylcholinesterase (AChE) and cholinacetyltransferase (ChAT) have been reported in $\mathrm{AD}$ brains $[13,20,21,37]$. It has also been described that in other dementias, such as parkinsonian dementia, cortical cholinergic function is even more severely affected than in $\mathrm{AD}$ [2].

Consequent to the cholinergic hypothesis of dementia, animal studies have been used to evaluate the role of cholinergic neurotransmission in learning and memory and selective lesion of cholinergic neurones remains nowadays as a widely used method to mimic some aspects of neurodegeneration in $\mathrm{AD}$ (see review [28]). In this sense, the most efficient tool to induce cholinergic hypoactivity is the immunotoxin 192 IgG-

\footnotetext{
* Corresponding author. Tel.: +34 948425600; fax: +34 948425649.

E-mail address: mariaja@unav.es (M.J. Ramírez).
}

saporin $[15,23,30]$. The verification and extent of the lesion are commonly assessed by post-mortem measuring of different biochemical markers, such as AChE [1,29], ChAT [24,33] or high-affinity choline uptake (HACU) [26]. All these parameters could be excellent markers of the extent of lesion or the amount of surviving cholinergic neurones subsequent to lesion. However, decreases in cholinergic markers may not reflect the activity of these remaining neurones due to the presence of compensatory mechanisms after long-term situations of cholinergic hypoactivity $[17,19]$. In addition, total acetylcholine (ACh) levels are labile in post-mortem tissue, and ACh released "in vivo" may not be detected in the absence of AChE inhibitors. Altogether, it is difficult to select the best cholinergic marker to study a situation of cholinergic deficit.

In the present work we sought to study the best marker of cholinergic hypoactivity in relationship to cognitive deficits. We have measured different cholinergic markers in two situations of cholinergic hypofunction: (1) in a human study, 
cholinergic function was assessed in post-mortem frontal cortex from $\mathrm{AD}$ patients who had been prospectively assessed with the mini-mental state examination (MMSE) for cognitive impairment and (2) in an animal study, cholinergic parameters were measured in the frontal cortex of rats subjected to a selective cholinergic lesion in the nucleus basalis magnocellularis (NBM). Statistical correlations between cholinergic and cognitive deficits have been also carried out.

Cholinergic functions was assessed in post-mortem frontal cortex (Brodmann area 10, BA10) of 22 patients with clinical diagnosis of dementia and 20 elderly normal controls matched for age, gender, post-mortem delay and brain $\mathrm{pH}$. Those patients with dementia were an autopsied subset of subjects included in a prospective study of behavioural changes in clinically diagnosed as demented patients [16]. Cognitive status was assessed at 4 monthly intervals using the mini-mental state examination (MMSE) [8].

Informed consent had been obtained from relatives before for removal of brain tissue at death. AChE, ChAT and total ACh levels in human brain tissues were measured as described by [11]. To partially mitigate the possible effects of cause of death on neurochemical determinations, brain $\mathrm{pH}$ was measured as an index of acidosis associated with terminal coma [18]. Post-mortem delay was also considered.

Male Wistar rats were kept at constant room temperature $\left(21 \pm 1^{\circ} \mathrm{C}\right)$ and relative humidity $(55 \pm 5 \%)$ with a 12 -h light/dark cycle (dark from 8 p.m.) and ad libitum access to food and water. A selective cholinergic lesion in the NBM was produced using the selective toxin $192 \mathrm{IgG}$-saporin. All the experiments were carried out in strict compliance with the recommendations of the EU (DOCE L 358/1 18/2/1986) for the care and use of laboratory animals. At the beginning of the study rats were allocated randomly to one of the experimental groups constituted by controls, sham-operated rats and rats with 192IgG-saporin lesions.

The production of the lesion was performed as described by [14]. Briefly, rats were anaesthetised with a mixture of ketamine $(10 \mathrm{mg} / \mathrm{kg}$, i.p.) and xylazine $(0.3 \mathrm{mg} / \mathrm{kg}$, i.p.). One microliters of the immunotoxin 192 IgG-Saporin $(0.067 \mu \mathrm{g} / \mu \mathrm{l} / \mathrm{hemisphere})$ was infused bilaterally into the nucleus basalis magnocellularis (NBM) of the basal forebrain at the following coordinates (from bregma): AP $-0.9 \mathrm{~mm}$, $\mathrm{ML} \pm 2.9 \mathrm{~mm}, \mathrm{DV}-6.5 \mathrm{~mm}$. Post-lesion survival times were established at 1 week and 1 month.

AChE activity, ChAT activity, total ACh levels, and ACh release both "in vivo" and "in vitro" were measured in the frontal cortex according to the methods described by [14].

Data were analysed by SPSS for Windows, release 11.0. Normality was checked by Shapiro-Wilks's test $(p>0.05)$. Student's $t$-test (human studies) or ANOVA test (animal studies) were used to study decreases in cholinergic parameters. The effects of demographic factors (age, post-mortem delay and brain $\mathrm{pH}$ ) on neurochemical variables, intercorrelation between neurochemical variables or relationships between severity of dementia (MMSE score at last interview before death) and neurochemical measures were determined by Pearson's or Spearman's rank correlation coefficients, according to the normality of variables. Significance was defined as $p \leq 0.05$.

The following compounds were used: ketamine (Ketasol, Parke-Davis, USA), xylazine (Rompun, Bayer, Germany) neostigmine bromide, hemicholinium-3, acetylthiocholine iodide (Sigma, UK); [methyl- $\left.{ }^{3} \mathrm{H}\right]$ choline chloride (86 Ci/mmol, PerkinElmer, USA); $\left[1-{ }^{14} \mathrm{C}\right]$ acetyl-coenzyme A $(59 \mathrm{mCi} / \mathrm{mmol}$, Amersham Biosciences, UK); 192IgG Saporin (lot number 21071083, Chemicon International, Inc., USA). Inorganic salts and other reagents were from Merck and Sigma.

Demographic details of subjects are shown in Table 1.

In controls, mean value for ACh levels, and AChE and ChAT activity were $0.36 \pm 0.04 \mathrm{nmol} / \mathrm{g}$ tissue, $99.75 \pm 2.80$ (absorbance) and $100.01 \pm 9.82 \mathrm{nmol} \mathrm{ACh} / \mathrm{h} g$ tissue, respectively. In the frontal cortex of $\mathrm{AD}$ patients, concentration of both ACh was significantly reduced (58\%) compared to controls. Similarly, AChE and ChAT activities in frontal cortex from AD patients were significantly lower (28.5 and $72.3 \%$, respectively) when compared to control values. ACh value, in $\mathrm{AD}$ patients, was significantly correlated with brain $\mathrm{pH}\left(n=22\right.$; Spearman's $\left.\rho=-0.861^{*} ; p=0.001\right)$ and postmortem delay $\left(n=22\right.$; Spearman's $\left.\rho=-0.503^{*} ; p=0.033\right)$. There were no significant correlations between brain $\mathrm{pH}$, post-mortem delay, length of tissue preservation time and AChE or ChAT activity Table 1.

In AD patients, cortical $\mathrm{AChE}$ and ChAT activities were significantly intercorrelated $\left(n=21 ; r=0.530^{*} ; p=0.019\right)$, while ACh levels did not correlate with either AChE or ChAT activities.

The mean MMSE score before death was $5 \pm 1$ in AD patients. A statistically significant positive correlation between last MMSE score and both AChE $(n=21$, Spearman's $\left.\rho=0.453^{*} ; p=0.045\right)$ and ChAT activities $(n=21$, Spearman's $\rho=0.473^{* *} ; p=0.030$ ) was found. However, ACh levels failed to show an association with the MMSE score.

In these studies, as there were no statistical differences and for simplicity purposes, control non-lesioned and sham animal data were combined in one same control group. Similarly, the two lesioned groups (corresponding to 1 week and 1 month post-lesion time) were combined in a single lesioned group. As expected, significant decreases were found in all cholinergic markers measured, as shown in Table 2.

Correlations between all cholinergic parameters are represented in Fig. 1. In the frontal cortex of animals with a selec-

Table 1

Demographic features of patients

\begin{tabular}{lll}
\hline & Control & Alzheimer \\
\hline Age (years) & $74.75 \pm 2.67$ & $81.06 \pm 1.60$ \\
Gender (male/female) & $11 / 9$ & $11 / 13$ \\
Post-mortem delay (h) & $39.28 \pm 5.40$ & $51.18 \pm 6.44$ \\
$\mathrm{pH}$ & $6.28 \pm 0.16$ & $6.42 \pm 0.54$ \\
\hline
\end{tabular}

$\mathrm{pH}$, standard chemical symbol, negative log of hydrogen ion concentration. (Control $n=19-20$, Alzheimer $n=20-22$ ). Values are mean \pm S.E.M. 
Table 2

Percentage of remaining activity/levels of different markers after cortical cholinergic dennervation

\begin{tabular}{llllll}
\hline Post-lesion time & AChE activity & ChAT activity & Total ACh levels & "In vivo" basal ACh levels & "In vitro" basal ACh levels \\
\hline 1 week & $52.89 \pm 4.25$ & $50.96 \pm 4.4$ & $45.91 \pm 4.20$ & $65.57 \pm 3.38$ & $43.33 \pm 1.88$ \\
1 month & $55.06 \pm 2.73$ & $54.99 \pm 3.93$ & $63.15 \pm 6.21$ & $67.52 \pm 3.51$ & $48.53 \pm 1.91$ \\
Total lesion & $54.21 \pm 2.93$ & $52.57 \pm 3.26$ & $52.63 \pm 3.88$ & $66.56 \pm 3.45$ & $46.24 \pm 1.80$
\end{tabular}

Data shown as mean \pm S.E.M. Percentage was calculated in relation to control and sham values. Control values were: AChE (absorbance) $=0.28 \pm 0.032$; $\mathrm{ChAT}=139.92 \pm 16.21 \mathrm{nmol} \mathrm{ACh} / \mathrm{h}$ g tissue; total $\mathrm{ACh}=3.82 \pm 0.73 \mathrm{nmol} / \mathrm{g}$ tissue; "in vivo" basal ACh levels $=464.15 \pm 24.76 \mathrm{fmol} / 15 \mu \mathrm{l}$; "in vitro" $\mathrm{ACh}$ levels $=2443.08 \pm 115.49 \mathrm{dpm}$. The two lesioned groups (corresponding to 1 week and 1 month post-lesion time) were combined in a single lesioned group (total lesion) for simplicity purposes, as there were no statistical difference between them.

tive cholinergic lesion, AChE activity significantly correlated with ChAT activity $\left(n=19\right.$; Pearson's $\left.r=0.556^{*} ; p=0.014\right)$, ACh levels $\left(n=18\right.$; Pearson's $\left.r=0.505^{*} ; p=0.033\right)$, and ACh release "in vivo" $\left(n=28\right.$; Spearman's $\rho=0.557^{*}$; $p=0.002$ ).

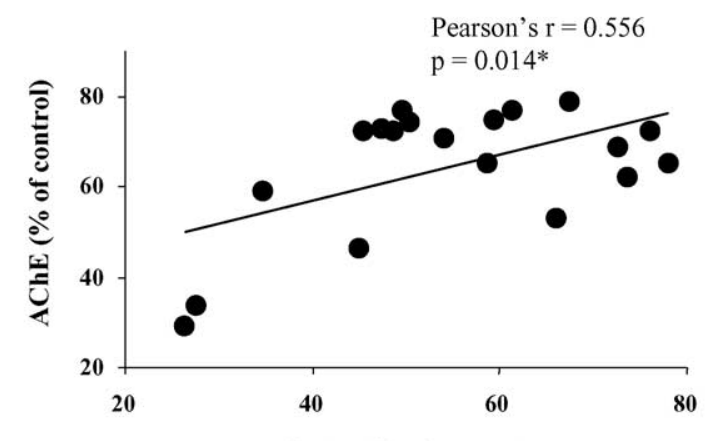

ChAT (\% of control)
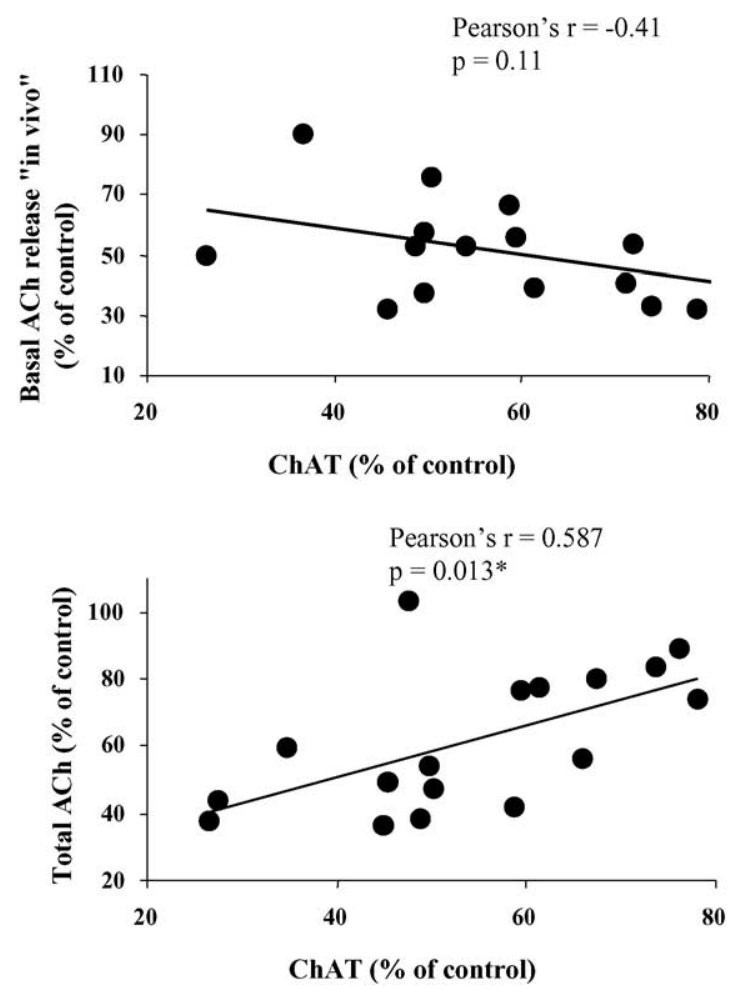

Disruption of basal forebrain cholinergic pathways and consequent cortical cholinergic denervation is one of the hallmarks of $\mathrm{AD}$, together with histopathological changes such as neurofibrilary tangles and senile plaques [20,21,37]. As expected, profound depletions in all cholinergic markers
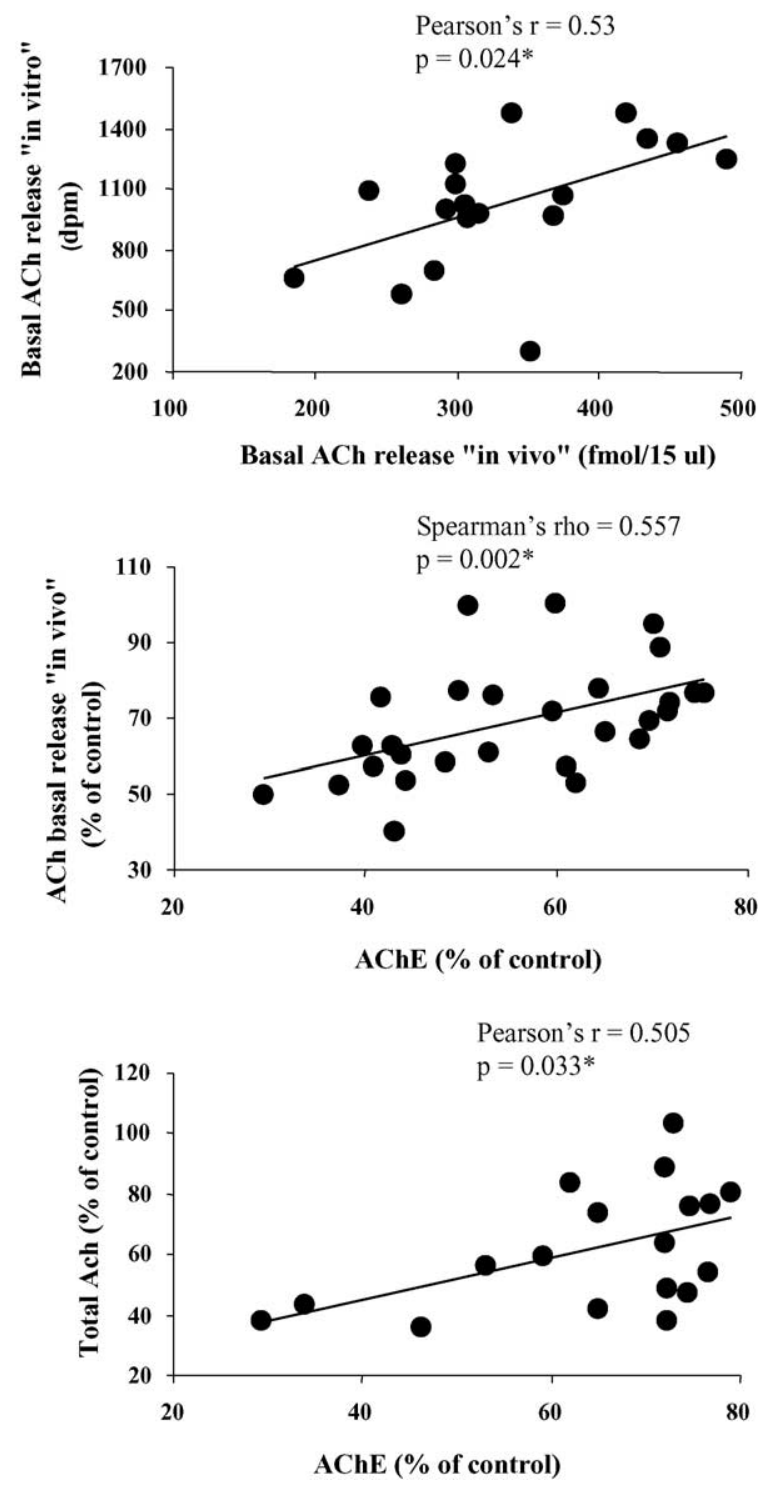

Fig. 1. Correlations between different cholinergic parameters measured in the frontal cortex of rats after a selective cholinergic lesion in the nucleus basalis magnocellularis. AChE, acetylcholinesterase; ChAT, coline acetylcholinesterase; ACh, acetylcholine. AChE activity is expressed as \% absorbance relative to controls; ChAT activity is expressed as nmol ACh/h g tissue; ACh levels are expressed as nmol/g tissue. 
measured (ACh, ChAT, AChE) were found in frontal cortex from $\mathrm{AD}$ patients in the present study. Data on total levels of $\mathrm{ACh}$, which are more directly related to neural activity, due to the degradation of ACh by $\mathrm{AChE}$ in post-mortem tissue, should be taken cautiously. Cholinergic degeneration in AD has been widely associated with cognitive impairments in the illness [28]. In our study not only ChAT, but also AChE levels in AD patients showed to be correlated to final MMSE scores. Decreases in AChE activity have also been described in several dementing conditions, such as parkinsonian dementia [2] or subcortical ischemic vascular dementia [34]. As the traditional treatment of $\mathrm{AD}$ are based on the use of AChE inhibitors, determination of the remaining AChE activity could be a good marker of the expected efficacy of the treatment to delay the progression of cognitive deficit. Supporting these data, $\mathrm{AChE}$ inhibitors such as rivastigmine and donezepil have been shown to maintain MMSE scores for up to 52 weeks in placebo-controlled study [27].

With respect to the animal studies, cholinergic neurones in the NBM give rise to a dense network of cholinergic fibres innervating the entire cortical mantle [32]. Partial cortical cholinergic deafferentiation was achieved by intraparenchymal infusion of the selective cholinergic immunotoxin, 192 IgG-Saporin, into the NBM $[15,30]$. It has been described that this immunotoxin, at the concentration used in the present work, produces maximal depletion of cortical cholinergic markers, sparing other non-cholinergic neuronal populations $[3,7,22,30]$. Under this experimental condition, a significant decrease in cholinergic function was achieved, as reflected by significant decreases in different cholinergic markers in the frontal cortex of lesioned animals, similar to those previously described after this type of lesion $[4,25,36]$. Furthermore, as expected, a decrease in "in vivo" [6] and "in vitro" [25] basal cortical ACh level was found.

ChAT activity has been long used as a marker of the loss of cholinergic neurones after a cholinergic lesion $[4,25,36]$. Since ChAT enzyme is not the rate-limiting step of ACh synthesis [31], it cannot be assumed that post-mortem assay of ChAT might be representative of the extracellular levels of $\mathrm{ACh}$, and therefore, of the cholinergic neural activity. There is a large body of evidence (e.g., [33]) that, at least 1 week after cholinergic lesion, surviving neurones may compensate the loss of terminals increasing the neural activity. If these compensatory mechanisms do not imply a regulation of some enzyme activities, then the post-mortem ChAT assay might be underestimating the real state of neurones after a cholinergic lesion. Accordingly to this, we did not find an association between ChAT activity and extracellular levels of ACh measured by microdialysis, thus, it appears to strengthen the hypothesis that ChAT may be a good marker for loss of cholinergic terminals but perhaps not for neural activity.

Traditionally, ChAT activity has been considered as the best marker of cholinergic function, as AChE appears to be located also in non-cholinergic post-synaptic neurones. However, in our study, decreases in ChAT and AChE activity seem to be correlated. Not only that, but AChE activity was cor- related to extracellular levels of $\mathrm{ACh}$, reinforcing $\mathrm{AChE}$, as an excellent candidate to evaluate the effects of a cholinergic lesion. The AChE activity assay is a fast, cheap and simple colorimetric method [5,35] compared to the radioenzymatic assay used to assess ChAT activity [9]. Thus, AChE activity assay described by [35] might be utilised as a rapid and first step assay to characterise a situation of cholinergic hypoactivity.

\section{Acknowledgements}

This work has been supported by a grant from Gobierno de Navarra (Spain) and through the "UTE project CIMA". F.J. Gil-Bea has a scholarship form Gobierno de Navarra (Spain). M. Garcia-Alloza has a scholarship from the Secretaria de Estado de Educacion y Universidades (Spain) co-funded by the Fondo Social Europeo (expte. EX2004-0250). The authors thank M.L. Muro and S. Lizaso for technical help.

\section{References}

[1] A. Birthelmer, A. Lazaris, C. Riegert, P. Marques-Pereira, J. Koenig, H. Jeltsch, R. Jackisch, J.C. Cassel, Does the release of acetylcholine in septal slices originate from intrinsic cholinergic neurons bearing p75(NTR) receptors? A study using 192 IgG-saporin lesions in rats, Neuroscience 122 (2003) 1059-1071.

[2] N.I. Bohnen, D.I. Kaufer, L.S. Ivanco, B. Lopresti, R.A. Koeppe, J.G. Davis, C.A. Mathis, R.Y. Moore, S.T. DeKosky, Cortical cholinergic function is more severely affected in parkinsonian dementia than in Alzheimer's disease: an in vivo positron tomographic study, Arch. Neurol. 60 (2003) 1745-1748.

[3] J.A. Burk, C.D. Herzog, M.C. Porter, M. Sarter, Interactions between aging and cortical cholinergic deafferentation on attention, Neurobiol. Aging 23 (2002) 467-477.

[4] S. de Lacalle, S. Kulkarni, R.G. Wiley, Lesion-induced transneuronal plasticity of the cholinergic innervation in the adult rat entorhinal cortex, Eur. J. Neurosci. 10 (1998) 1054-1062.

[5] G.L. Ellman, K.D. Courtney, V.J. Andres, R.M. Feather-Stone, A new and rapid colorimetric determination of acetylcholinesterase activity, Biochem. Pharmacol. 7 (1961) 88-95.

[6] J. Fadel, H. Moore, M. Sarter, J.P. Bruno, Trans-synaptic stimulation of cortical acetylcholine release after partial 192 IgG-saporininduced loss of cortical cholinergic afferents, J. Neurosci. 16 (1996) $6592-6600$

[7] I. Ferencz, G. Leanza, A. Nanobashvili, M. Kokaia, O. Lindvall, Basal forebrain neurons suppress amygdala kindling via cortical but not hippocampal cholinergic projections in rats, Eur. J. Neurosci. 12 (2000) 2107-2116.

[8] M.F. Folstein, S.E. Folstein, Mini-mental state. A practical method for grading the cognitve state of patients for the clinician, J. Psychiatr Res. 12 (1975) 189-198.

[9] F. Fonnum, A rapid radiochemical method for the determination of choline acetyltransferase, J. Neurochem. 24 (1975) 407-409.

[10] P.T. Francis, A.M. Palmer, M. Snape, G.K. Wilcock, The cholinergic hypothesis of Alzheimer's disease: a review of progress, J. Neurol. Neurosurg. Psychiatry 66 (1999) 137-147.

[11] M. Garcia-Alloza, W.D. Hirst, C.P. Chen, B. Lasheras, P.T. Francis, M.J. Ramirez, Differential involvement of 5-HT(1B/1D) and 5-HT6 receptors in cognitive and non-cognitive symptoms in Alzheimer's disease, Neuropsychopharmacology 29 (2004) 410-416. 
[12] C. Geula, M.M. Mesulam, Cholinergic systems and related neuropathological predilection patterns in Alzheimer's disease, in: K.L. Bick, R. Katzman, R.D. Terry (Eds.), Alzheimer Disease, Raven Press, New York, 1994, pp. 263-303.

[13] E. Giacobini, Cholinergic function and Alzheimer's disease, Int. J. Geriatr. Psychiatry 18 (2003) S1-S5.

[14] F.J. Gil-Bea, J. Dominguez, M. Garcia-Alloza, B. Marcos, B. Lasheras, M.J. Ramirez, Facilitation of cholinergic transmission by combined treatment of ondansetron with flumazenil after cortical cholinergic deafferentation, Neuropharmacology 47 (2004) 225-232.

[15] S. Heckers, T. Ohtake, R.G. Wiley, D.A. Lappi, C. Geula, M.M. Mesulam, Complete and selective cholinergic denervation of rat neocortex and hippocampus but not amygdala by an immunotoxin against the p75 NGF receptor, J. Neurosci. 14 (1994) 1271-1289.

[16] T. Hope, J. Keene, C. Fairburn, R. McShane, R. Jacoby, Behavior changes in dementia 1: Point of entry data of a prospective study, Int. J. Geriatr. Psychiatry 12 (1997) 1062-1073.

[17] P.A. Lapchak, D.J. Jenden, F. Hefti, Compensatory elevation of acetylcholine in vivo by cholinergic neurons surviving partial lesions of the septohippocampal pathway, J. Neurosci. 9 (1991) 2121-2128.

[18] D.A. Lewis, The human brain revisited: opportunities and challenges in post-mortem studies of psychiatric disorders, Neuropsychopharmacology 26 (2002) 143-154.

[19] J. McGaughy, J.W. Dalley, C.H. Morrison, B.J. Everitt, T.W. Robbins, Selective behavioral and neurochemical effects of cholinergic lesions pruduced by intrabasalis infusions of 192 IgG-Saporin on attentional performance in a five-choice serial reaction time task, J. Neurosci. 22 (2002) 1905-1913.

[20] P.L. McGeer, E.G. McGeer, J. Suzuki, C.E. Dolman, T. Nagai, Aging, Alzheimer's disease, and the cholinergic system of the basal forebrain, Neurology 34 (1984) 741-745.

[21] J. Pascual, A. Fontan, J.J. Zarranz, J. Berciano, J. Florez, A. Pazos, High-affinity choline uptake carrier in Alzheimer's disease: implications for the cholinergic hypothesis of dementia, Brain Res. 552 (1991) 170-174.

[22] T. Perry, H. Hodges, J.A. Gray, Behavioural, histological and immunocytochemical consequences following 192 IgG-saporin immunolesions of the basal forebrain cholinergic system, Brain Res. Bull. 54 (2001) 29-48.

[23] D.P. Pizzo, J.J. Waite, L.J. Thal, J. Winkler, Intraparenchymal infusions of $192 \mathrm{IgG}$-saporin: development of a method for selective and discrete lesioning of cholinergic basal forebrain nuclei, J. Neurosci. Methods 91 (1999) 9-19.

[24] L. Ricceri, C. Hohmann, J. Berger-Sweeney, Early neonatal $192 \mathrm{IgG}$ saporin induces learning impairments and disrupts cortical morphogenesis in rats, Brain Res. 954 (2002) 160-172.
[25] S. Rossner, R. Schliebs, W. Hartig, V. Bigl, 192IgG-saporin-induced selective lesion of cholinergic basal forebrain system: neurochemical effects on cholinergic neurotransmission in rat cerebral cortex and hippocampus, Brain Res. Bull. 38 (1995) 371-381.

[26] S. Rossner, J. Yu, D. Pizzo, K. Werrbach-Perez, R. Schliebs, V. Bigl, J.R. Perez-Polo, Effects of intraventricular transplantation of NGFsecreting cells on cholinergic basal forebrain neurons after partial immunolesion, J. Neurosci. Res. 45 (1996) 40-56.

[27] P.N. Tariot, Maintaining cognitive function in Alzheimer disease: how effective are current treatments, Alzheimer Dis. Assoc. Disord. 15 (2001) S26-S33.

[28] A.V. Terry, J.J. Buccafusco, The cholinergic hypothesis of age and Alzheimer's disease-related cognitive deficits: Recent challenges and their implications for novel drug development, J. Pharmacol. Exp. Ther. 306 (2003) 821-822.

[29] M. Tomaszewicz, S. Rossner, R. Schliebs, J. Cwikowska, A. Szutowicz, Changes in cortical acetyl-CoA metabolism after selective basal forebrain cholinergic degeneration by 192IgG-saporin, J. Neurochem. 87 (2003) 318-324.

[30] E.M. Torres, T.A. Perry, A. Blokland, L.S. Wilkinson, R.G. Wiley, D.A. Lappi, S.B. Dunnett, Behavioural, histochemical and biochemical consequences of selective immunolesions in discrete regions of the basal forebrain cholinergic system, Neuroscience 63 (1994) 95-122.

[31] S. Tucek, Short-term control of the synthesis of acetylcholine, Prog. Biophys. Mol. Biol. 61 (1993) 59-69.

[32] B.H. Wainer, M.M. Mesulam, Ascending cholinergic pathways in the rat brain, in: M. Steriade, D. Biesold (Eds.), Brain Cholinergic Systems, Oxford University Press, Oxford, 1990, pp. 65-119.

[33] J.J. Waite, A.D. Chen, Differential changes in rat cholinergic parameters subsequent to immunotoxic lesion of the basal forebrain nuclei, Brain Res. 918 (2001) 113-120.

[34] A. Wallin, M. Sjogren, K. Blennow, P. Davidsson, Decreased cerebrospinal fluid acetylcholinesterase in patients with subcortical ischemic vascular dementia, Dement. Geriatr. Cogn. Disord. 16 (2003) 200-207.

[35] H. Wang, P.R. Carlier, W.L. Ho, D.C. Wu, N.T.K. Lee, C.P.L. Li, Y.P. Pang, Y.F. Han, Effects of bis(7)-tacrine, a novel anti-Azlheimer's agent, on rat brain AChE, NeuroReport 10 (1999) 789-793.

[36] G.L. Wenk, J.D. Stoehr, G. Quintana, S. Mobley, R.G. Wiley, Behavioral, biochemical, histological, and electrophysiological effects of 192 IgG-saporin injections into the basal forebrain of rats, J. Neurosci. 14 (1994) 5986-5995.

[37] P.J. Whitehouse, D.L. Price, R.G. Struble, A.W. Clark, J.T. Coyle, M.R. Delon, Alzheimer's disease and senile dementia: loss of neurons in the basal forebrain, Science 215 (1982) 1237-1239. 